\title{
P 231 INTEGRATED PALLIATIVE CARE/ADVANCED RESPIRATORY DISEASE (ARD) CLINICS - A MULTI-CENTRE PILOT PROJECT
}

Claire Curtis, ${ }^{1}$ Simon Challand, ${ }_{1}^{1}$ Susan Harrison, ${ }^{2}$ Marion Turner, ${ }^{3}$

Elaine Bevan-Smith ${ }^{4}$. ${ }^{1}$ Worcestershire Health and Care NHS Trust; ${ }^{2}$ KEMP Hospice, Kidderminster; ${ }^{3}$ St Richards Hospice, Worcester; ${ }^{4}$ Worcestershire Acute Hospitals NHS Trust

10.1136/bmjspcare-2014-000654.272

Background The symptom burden of ARD is similar to that of cancer, however patients with COPD are less likely to access Specialist Palliative Care (SPC) Services, and less likely to die at home. In Worcestershire there is a comprehensive COPD service providing an ARD pathway, but there exists a group of patients with ARD, who are too unwell to attend the pulmonary rehabilitation and fit to breathe groups, who need a differing level of support, but who are reluctant to attend generic hospice day care. Aims The monthly joint out-patient clinics aim to enhance end of life care for patients with ARD and their carers by:

- Providing specialist medical and nursing input from Respiratory and Palliative Medicine consultants, COPD CNS, and SPC day hospice team to minimise symptoms, and maximise activity and function.

- Improving joint working and increasing accessibility and acceptability of SPC services.

- Challenging and changing the culture and aims of care for ARD patients.

- Reducing un-planned hospital admissions.

- Offering ACP

Results Monthly clinics were set up at two hospice sites, and have been running for 18 months.

Approx 50 patients have attended.

Unplanned hospital admissions have reduced, deaths at home have increased. 
Patient and Carer satisfaction survey, HADS and distress thermometer have been used.

ACP has been undertaken using the Worcestershire advance statement document.

Conclusions The clinics have evolved over the 18 months and benefits have occurred beyond just the scope of the patients who actually attend in person. There exists the ability to offer another treatment pathway, with culture change occurring because of this extra option for care. Teams have been empowered to engage in ACP, there has been enhanced carer support and improved joint working. 